\title{
Control of Visual Selection during Visual Search in the Human Brain
}

\author{
Manuel C. Olma \\ Department of Neurology, Charité, Humboldt-University of Berlin \\ Tobias H. Donner \\ New York University \\ Stephan A. Brandt \\ Humboldt-University of Berlin
}

\begin{abstract}
How do we find a target object in a cluttered visual scene? Targets carrying unique salient features can be found in parallel without directing attention, whereas targets defined by feature conjunctions or non-salient features need to be scrutinized in a serial attentional process in order to be identified. In this article, we review a series of experiments in which we used fMRI to probe the neural basis of this active search process in the human brain. In all experiments, we compared the fMRI signal between a difficult and an easy visual search (each performed without eye movements) in order to isolate neural activity reflecting the search process from other components such as stimulus responses and movementrelated activity. The difficult search was either a conjunction search or a hard feature search and compared with an easy feature search, matched in visual stimulation and motor requirements. During both, the conjunction search and the hard feature search the frontal eye fields (FEF) and three parietal regions located in the intraparietal sulcus (IPS) were differentially activated: the anterior and posterior part of the intraparietal sulcus (AIPS, PIPS) as well as the junction of the intraparietal with the transverse occipital sulcus (IPTO). Only in PIPS, the modulation strength was most indistinguishable between conjunction and hard feature search. In a further experiment we showed that AIPS and IPTO are involved in visual conjunction search even in the absence of distractors; by contrast, the involvement of PIPS seems to depend on the presence of distractors. Taken together, these findings from these experiments demonstrate that all four key nodes of the human 'frontoparietal attention network' are generally engaged in the covert selection process of visual search. But they also suggest that these areas play differential roles, perhaps reflecting different sub-processes in active search. We conclude by discussing a number of such sub-processes, such as the direction of spatial attention, visual feature binding, and the active suppression of distractors.
\end{abstract}

\section{Keywords:}

fMRI, Frontal eye fields, Posterior parietal cortex, visual attention, visual search

\section{Natural vision and active selection}

Constantly, natural visual scenes confront us with a multitude of visual information, only few of which is relevant for our actions such as eye or grasping movements. Therefore selective visual attention plays a central role in the control of our everyday behavior. One essential contribution that shed light on the function of visual attention was L. Stark's work. He recorded subjects' eye movements during free-viewing of a visual scene (Noton \& Stark, 1971, see Fig1).

One recognizes that visual exploration consists of a cycle of fixation periods and saccades. During periods of fixation, the following processes take place: 1. perceptual processing and identification of the fixated object. 2. selection of potential fixation locations. 3. preparation and execution of the saccade. The second process is frequently equated with visual attention. (Andersen \& Gnadt, 1989; Colby \& Goldberg, 1999; Schall \& Thompson, 1999). Since object selection is a prerequisite for the execution of all visually controlled actions, visual attention can be defined more generally as the selection of visual representations for the access to the control of action (Duncan \& Humphreys, 1989; Allport, 1993; Desimone \& Duncan, 1995). Specifying the types of representations which serve as a basis for attentional selection has 
for long been an active area of attention research (Treisman and Gelade, 1980; Cave and Bichot, 1999; Kastner and McMains, 2007). It now seems clear that the units of attentional selection can be not only locations in space (Posner, 1980; Treisman and Gelade, 1980; Bichot and Desimone, 2006), but also single features (Bichot and Desimone, 2006; Maunsell and Treue, 2006), and even unified objects (Duncan, 1984; O'Craven et al, 1999; Blaser et al, 2000; Mitchell et al, 2004).

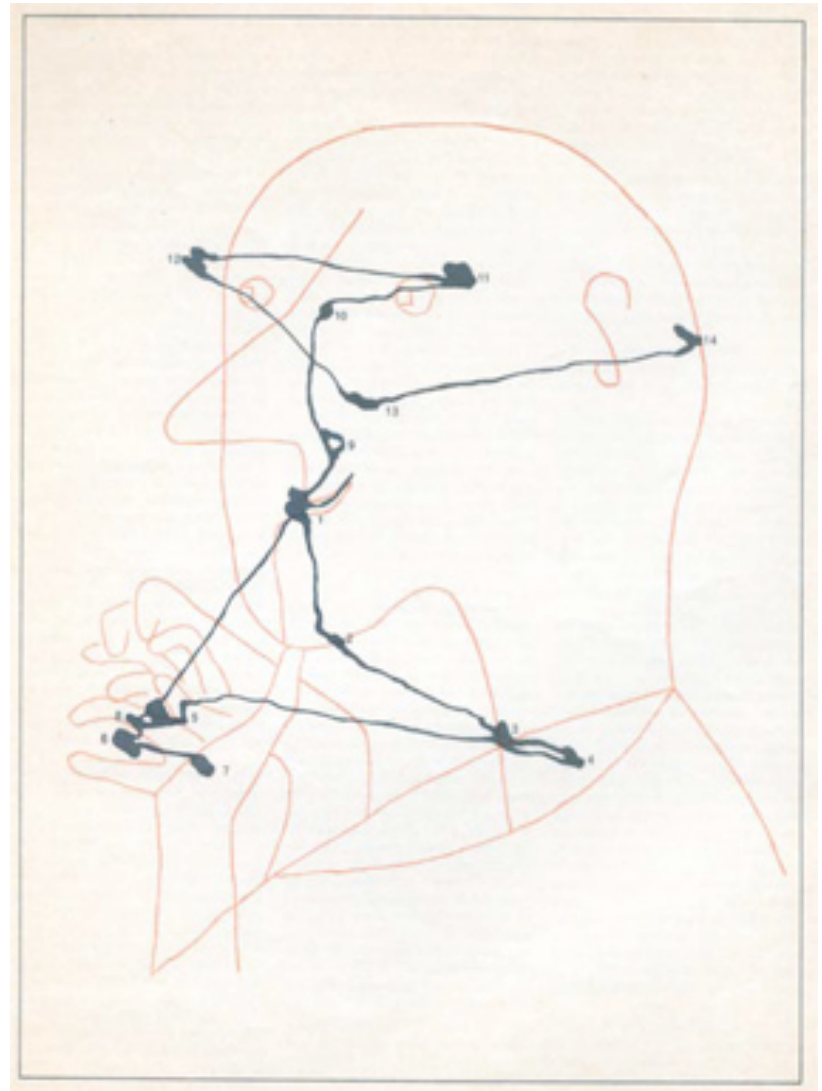

Figure 1. Eye movements made by a subject viewing for the first time a drawing adapted from Paul Klee's "Old Man Figuring" appear in black. Numbers show the order of the subject's visual fixations on the picture during part of a 20-second viewing. Lines between them represent saccades from one fixation to the next. Saccades occupy about 10\% of viewing time. (from Noton \& Stark, 1971, Scientific America)

\section{The visual search paradigm}

Visual selection processes underlying object recognition in complex scenes have been extensively characterized on the behavioral level; the experimental paradigm is commonly referred to as visual search (see Wolfe, 1998, for a review). In these experiments the everyday process of visual search was mimicked by instructing subjects to search for a target object within an array of visual objects. In some studies, the exploration of the stimulus array by eye movements was allowed. In these studies, reaction times and the pattern of eye movements were observed (e.g. Bichot \& Schall, 1999, Findlay, 1997; Groner \& Groner 2000). In other studies, however, restricted inspection conditions were used, i.e., the subject was instructed not to move eyes during search (Wolfe, 1998). This procedure utilized the subject's ability to direct attention to objects in the periphery of the visual field while fixating a central point. This ability to shift attention was also termed covert attention (Posner \& Petersen, 1990). During visual search the target was present in some trials while only distractors appeared in others. The subject had to decide whether the target was present or not. The amount of the objects in the array was varied (i.e. variable set sizes) and reaction times were depicted as a function of set size. This function was fitted linearly by a regression analysis and briefly named "search slope". If the search slope ran flat, reaction times were independent of set size. If it increased, the presence of each further object cost time. In general, the slope of the search function is understood as a measure of difficulty of the according search task, independently of the postulated selection processes underlying the slope (Wolfe, 1998).

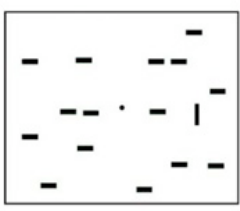

Feature search

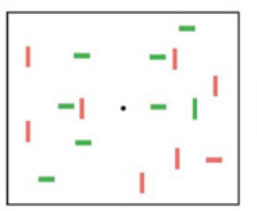

Conjunction search

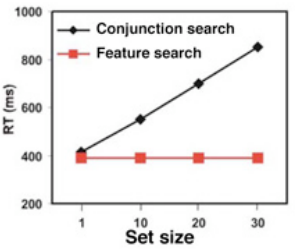

Figure 2. The visual search paradigm. Left: stimulus array of a typical feature search. Middle: stimulus array of a typical conjunction search. Right: idealized result pattern

In the left stimulus array of figure 2 , the vertical target bar differs from the remaining bars by its orientation (as an elementary visual feature). This type of search was termed feature search. In the middle stimulus array, the target is a vertical-green bar amongst vertical-red and horizontal-green bars. In this case, the target differs from the distractors only in the conjunction of its features. That type of search was named conjunction search. On the right side, idealized search slopes are depicted that resulted from experiments of this type in classic studies (Treisman \& Gelade, 1980). The greater slope of the black curve indicates that the conjunction search is more difficult than the feature search. This basic result pattern 
was replicated in a numerous studies (Wolfe, 1998; Cave \& Wolfe, 1999).

Later studies, however, revealed striking deviations from this typical pattern: First, some conjunction searches produced flat search functions (Nakayama and Silverman, 1986). Second, feature search behavior exhibited a strong dependence on attention (Joseph et al, 1997). These and other results indicated that conjunction and feature search foot on uniform selection processes and differ only quantitatively (Duncan \& Humphreys, 1989; Wolfe, 1998). Selection efficiency was argued to depend on the similarity between target and distractors (Duncan \& Humphreys, 1989). Target and distractors are more similar in conjunction search than in feature search, as only in the former, distractors share common features with the target (see Fig 2).

\section{Functional models of visual search}

According to classic-serial models (Treisman \& Gelade, 1980; Koch \& Ullmann, 1985), the visual system initially analyzes the whole scene by extracting its elementary features (e.g. color or orientation of contours). Subsequently, these features are integrated to objects by focusing attention on a location. Specifically, Treisman and Gelade's (1980) classical feature integration theory explained the apparent behavioral dichotomy between efficient feature search and inefficient conjunction search with a dichotomy of search mechanisms: Feature search is based on the detection of a unique feature in one of the topographic maps representing visual features; a target with a unique feature does not need to be integrated, and hence, can be found without the employment of attention. By contrast, focal attention is required to integrate visual features into representations of objects. Therefore, single positions of the scene have to be selected serially by focal attention in order to find a target defined by a feature conjunction. This theory was attractive because strong link to the multiple parallel 'feature maps' in the primate visual cortex (Felleman and VanEssen, 1991; Treisman, 1996). The 'master map' of locations controlling spatial selection could be naturally mapped onto spatial representations in posterior parietal cortex (PPC; Treisman, 1996; Colby \& Goldberg, 1999). But it could not account for the efficient conjunction searches and inefficient feature searches observed in later behavioral studies.

Subsequent models postulated a common selection process for feature and conjunction searches operating in parallel by simultaneously evaluating the behavioral relevance of all objects in the visual scenes (Duncan \& Hum- phreys, 1989; Desimone \& Duncan, 1995; Usher \& Niebuhr, 1996). Duncan and Humphrey's (1989) "Attentional Engagement Theory" is based on the assumption that search performance is explained by both the (dis-) similarity between targets and distractors and the (dis-) similarity among the distractors. Search difficulty depends on the discriminability of target and distractors, and not on the necessity of feature conjunction. All objects in the scene compete for 'weights' controlling a limitedcapacity short- term memory stage controlling behavior (see also Duncan et al, 1997). Crucially, selection in this and related models is guided by a memory representation of the searched-for target, which is now commonly assumed to be stored in the prefrontal cortex (PFC; Desimone and Duncan, 1995; Usher \& Niebuhr, 1996).

In more recent parallel-serial hybrid models, such as Wolfe's (1994) "Guided Search" (see also Bichot and Desimone, 2006), the selection process ultimately operates serially, but under the parallel guidance of a memory representation of the target features ('top-down') and a representation of the conspicuousness of local features ('bottom-up'); These two are combined into a topographic representation of visual salience indicating the behavioral relevance of local stimuli (Treue 2003). Such a representation has been proposed to exist in the PPC (Gottlieb et al, 1998) and the frontal eye fields (FEF; Bichot et al, 1999; Thompson and Bichot, 2005). During visual search focal attention is directed to the most salient location first, then to the second most salient location, etc. The top-down guidance is the more difficult, the less the target is discriminable from distractors. A large-scale neural network model motivated by these hybrid theories (Hamker, 2004) focuses on the 're-entry' of spatial attention signals in a distributed network and on how this process can be guided by memorized features. This model predicts that attention builds up successively by convergence and feedback. Feedback passes the target information from PFC to IT and extrastriate areas as V4. The "oculomotor circuit", comprising FEF, LIP and superior colliculus, includes this distributed activity and yields a continuous spatial reentry signal.

Thus, these three broad classes of functional models make different predictions regarding the cortical substrates involved in the selection mechanisms of visual search. Parallel models predict that the memory representation of the target, stored in the PFC directly controls the selection in visual search, operating on representations in the visual cortices. By contrast, if spatial locations are selected serially, as claimed by the classic serial and hy- 
brid models, the PPC (and FEF) must be engaged in the processes that control visual search.

\section{Probing the neural correlates of covert visual search in the human brain}

Functional neuroimaging is ideally suited for addressing these predictions because it allows for simultaneously monitoring the activity of these widely distributed cortical association areas while human subjects perform a variety of visual search tasks designed to isolate the selection processes discussed above. Studying the neural basis of attentional selection during visual search might also provide deeper insights into important general principles underlying the control of visually-guided orienting behavior by large-scale cortical networks. Therefore, our and several other laboratories began to address these questions using fMRI a few years ago.

These studies could build on the fact that the neural correlates of visuo-spatial attention had been already been studied extensively in the human brain since the emergence of functional neuroimaging (Posner and Peterson, 1990; Corbetta et, 1998; Kastner and Ungerleider, 2000). These studies agreed that FEF and the PPC are involved in overt (i.e. accompanied by eye movements) as well as in covert shifts of attention (Corbetta et al, 1998). More recent fMRI studies (Corbetta et al, 1998) with a precise analysis of single subjects showed that three separate sub-regions in the intraparietal sulcus (IPS) can be dissociated: the anterior part of the intraparietal sulcus (AIPS); the posterior part of the intraparietal sulcus (PIPS) and the junction of the intraparietal with the transverse occipital sulcus (IPTO).

\section{A frontoparietal network for covert visual search}

In a first experiments (Donner et al., 2000), we therefore tested if the FEF and these three intraparietal subregions are also involved in visual conjunction search, as predicted by classical and hybrid models involving a spatially serial selection process. In the experimental condition, subjects searched for a target defined by a conjunction of color and orientation (conjunction search, see Fig $3)$. In the baseline condition (easy feature search), subjects searched for a uniquely colored target, regardless of its orientation. In order to minimize the occurrence of saccadic eye movements in the scanner, the search arrays were masked after the presentation time of $80 \mathrm{~ms}$.

Reaction times increased significantly with set size both in conjunction search and but not in easy feature search. This indicated that the experimental condition conjunction search was attentionally more demanding than the control condition easy feature search (see Fig 3).

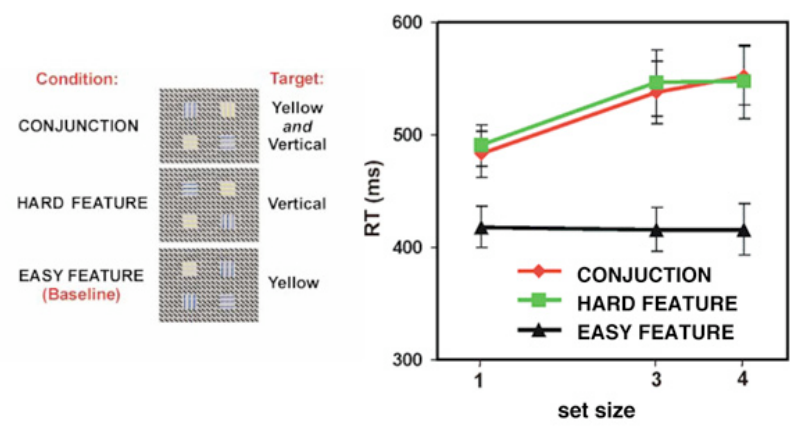

Figure 3. Left panel: Visual search conditions. Exemplary "target-present trials" are shown for the Hard Feature, Easy Feature, and Conjunction tasks. The target cluster consisted of vertical bars of either color in Hard Feature (lower right quadrant), yellow bars of either orientation in Easy Feature (upper left quadrant), and of yellow and vertical bars in Conjunction (upper right quadrant). Subjects were instructed to fixate and to indicate the absence or presence of the target. Due to the higher luminance of the yellow clusters, the ratio of target to distracter salience was lower in Hard Feature, rendering search more difficult than in Easy Feature.

Right panel: search slopes for conjunction search, hard feature search and easy feature search. Reaction times from psychophysical validation are depicted as function of set size. Values are means of correct responses. The error bars indicate the standard error.

Contrasting both conditions (conjunction search and easy feature search), significant differential activations between conditions were ascribed to top-down modulation of neural activity (see Fig 4). The activated region in the dorsal part of the precentral sulcus corresponds unambiguously in its localization to the human analogue of FEF (Corbetta; 1998; Paus, 1996; Courtney et al., 1998; Beauchaump et al., 2001). FEF and PPC were consistently activated. PPC showed activation with a larger extent on the right hemisphere. In principle, this is in line with the assumption of the predominance of the right hemisphere for the control of spatial attention (Mesulam, 1999). However, it indicates a quantitative rather than a qualitative difference of the contribution of both PPCs. The group activation showed three statistical peaks in the intraparietal sulcus corresponding to the three sub-regions AIPS, PIPS and IPTO. The anatomical position and the talairach coordinates von AIPS, PIPS and IPTO agree well with the three sub-areas found by Corbetta et al. (1998) during spatial shifts of attention. For the group analyses, individual brains needed to be transformed in a 
standardized brain, which reduces spatial discriminatory power. This leads to largely fused activations in PPC. Therefore a multi regression analysis was performed that was based not on a group, but on individual data. As a result, consistent over the subjects, activations in the parietal regions were spatially distinct (see Fig 4). These results suggest an involvement of the human frontal eye field in covert visual selection of potential targets during search. The activation of the right posterior parietal cortex was roughly consistent with a the results of an earlier PET study of visual search using similar stimulus array (Corbetta et al. 1995), in which a conjunction search task was also contrasted with an easy feature task. However, Corbetta et al. (1995) described no task-related modulation in the FEF. Moreover, surface reconstruction and analysis of individual subjects permitted more finegrained mapping of multiple distinct parietal activations in our study. The activation site in the right superior parietal lobe observed by Corbetta et al. (1995) seemed to correspond best to the right posterior IPS region.

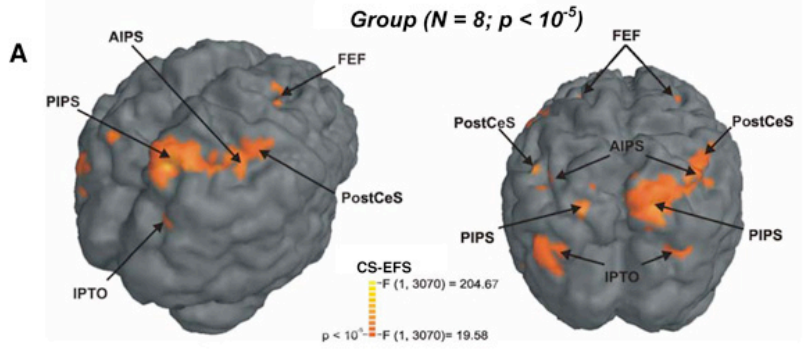

B

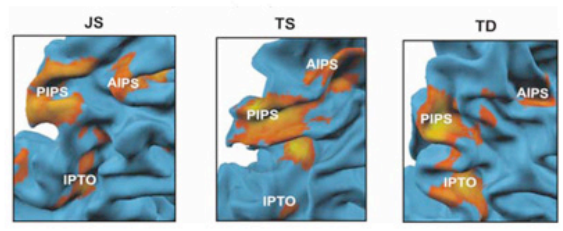

Figure 4. A) Group activation map, superimposed on one subject's rendered brain. Left, dorsolateral view; right, dorsoposterior view. Activations are produced by conjunction search (CS), relative to easy feature search (EFS).

Abbreviations: PreCeS, precentral sulcus; PostCeS, postcentral sulcus; AIPS, anterior intraparietal sulcus; PIPS, posterior intraparietal sulcus; IPTO, intraparietal transverse occipital sulcus. Posterior parietal activation is larger in the right than in the left hemisphere, but contains corresponding peaks in both hemispheres. B) Sections of folded posterior parietal cortex of three exemplary subjects with their individual activation patterns. PIPS consistently extends to the convexity of the superior parietal lobulus.

\section{Feature binding is not critical for engaging the frontoparietal network in visual search}

What type of attentional process does this activation pattern reflect? It had been proposed that the engagement of parietal cortex in object identification is linked to the process of feature conjunction through spatial selection (Friedman-Hill et al, 1995; Treisman, 1996; Robertson, 1998). We wondered whether the necessity to conjoin the features (i.e. color and orientation) for target identification is a prerequisite for the involvement of PPC as well as FEF in attentive visual search. Besides imposing the need for feature integration, visual conjunction searches are also more difficult than many simple feature searches because the targets are commonly of lower relative saliency as compared to the distractors (Wolfe et al, 1994, Wolfe, 1998). We therefore investigated in a second experiment (Donner et al., 2002), whether high search difficulty alone, without the necessity for feature integration, is sufficient for a frontoparietal engagement in visual search.

To this end, we introduced a new experimental condition: hard feature search. By means of an adequate psychophysical manipulation, this new condition was adjusted to the difficulty of conjunction search (see Fig 3). In order to render hard feature search more difficult than easy feature search, the ratio of target and distracter salience had to be decreased in hard feature search by a modulation of the objects' luminance. Thus, the difference between experimental and control condition reflects only difficulty and not a combination of difficulty and feature conjunction (as in the first experiment). If the frontoparietal areas are differentially activated, their activity correlates generally with difficulty, as all other factors (sensory stimulation and motor response) are identical.

Moreover, both differential responses (conjunction search - easy feature search; and hard feature search easy feature search) can be assessed by a direct quantitative comparison. This comparison allows for testing whether there are areas that show equally strong activation, which is correlated only with difficulty and not with feature conjunction. For that purpose, regions in FEF, AIPS, PIPS and IPTO, activated during conjunction search, were identified on cortical surfaces and served as regions of interest (ROI) (see Fig 5).

In each of the four ROIs, the fMRI signal was relative to the common control condition (easy feature search). Furthermore, the fMRI signal was averaged over all voxels of the ROI, over all trials of each condition, over both 
hemispheres and over all subjects. The resulting means were compared statistically. The amplitude of the fMRI signal modulation in AIPS was higher during hard feature search than during conjunction search, whereas the modulation in FEF and IPTO was stronger during conjunction search. There was no difference between conditions in PIPS.
A
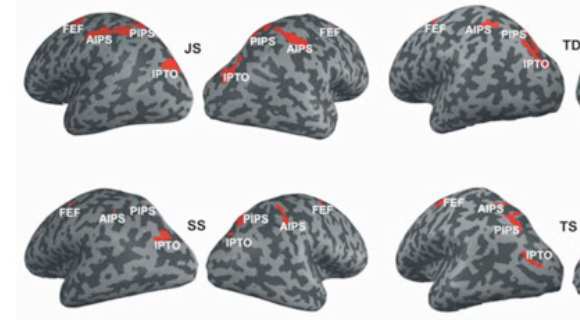

B

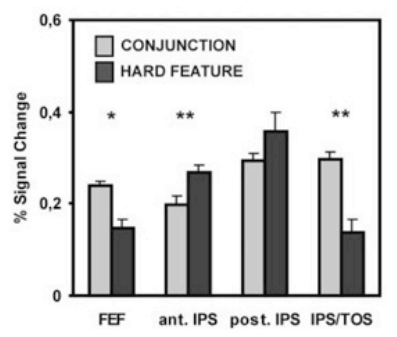

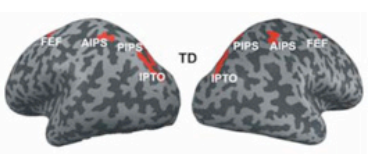

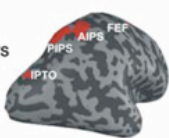

Figure 5. A) ROIs for the comparision of the fMRI responses in conjunction search and hard feature search. Significant activated regions during conjunction search served as ROIs and were marked in red on the enfolded cortical surfaces. B) FMRI responses during Hard Feature and Conjunction. Normalized and averaged response amplitudes of the regions in FEF, AIPS, PIPS and IPTO of significant activation during Conjunction are noted for Conjunction and Hard Feature. Error bars represent standard error. Significant differences between Conjunction and Hard Feature are indicated by "*" for $P, 0.05$ and by "**" for $P$, 0.01. Abbreviations: FEF, frontal eye field; AIPS, anterior intraparietal sulcus; PIPS, posterior intraparietal sulcus; IPTO, intraparietal transverse occipital sulcus.

The activity of PIPS was tightly correlated with search difficulty. This finding supports the hypothesis that this area contains the human analogue of LIP of the macaque (Corbetta, 1998; Corbetta et al., 1998). Based on physiological evidence, there is presumably a salience map implemented in LIP (Gottlieb et al., 1998; Itti \& Koch, 2001) on which, according to spatial serial models, spatial selection takes place (Wolfe, 1994). The activity of LIP during search should reflect differences in difficulty. Furthermore, the diverse pattern of modulation of AIPS, PIPS and IPTO during both search condition indi- cates that PPC sub-regions incorporate different functional properties.

To sum up, conjunction search and hard feature search were not discriminable in their psychophysical characteristics during the psychophysical validation. The absent difference in reaction times between conjunction search and hard feature search at a set size of four objects during the two experiments is in line with the psychophysical validation. In the second experiment, similar areas as in the first experiment were activated, in particular FEF, AIPS, PIPS and IPTO. Analogue to the first experiment, the results indicate the bilateral involvement of PPC during search with a gradual asymmetry in favor of the right hemisphere. In particular, this result seems to be contradictory to the common hypothesis that the activation of PPC during visual search reflects the process of feature conjunction specifically. However, the strength of activation was only not significantly different in PIPS during conjunction search and hard feature search. The response of this region reflects the same difficulty of both tasks in the clearest way. By means of further control experiments, it was excluded that the differential activations in the two experiments reflected a difference of the deployment of eye movements or the minimal differences of the stimuli between experimental and control condition. Overall, the results show that the differential activations in the two experiments reflect neither sensory nor motor processes but rather visual selection processes.

Nonetheless, PPC engagement is a very general feature of a difficult visual task (Wojciulik \& Kanwisher, 1999, Culham \& Kanwisher, 1999). Two mechanisms have been proposed to account for this general role: (1) PPC is a source of top-down signals counteracting suppressive effects of distractors on the target, thereby biasing object competition towards the target (Reynolds \& Desimone, 1999). (2) PPC actively inhibits distractors (Wojciulik \& Kanwisher, 1999). The common characteristic of both hypotheses is the critical significance of the presence of multiple distractors for a PPC involvement in visual tasks. This leads to the following prediction:

- Parietal cortex should not be engaged in visual search in the absence of distractors in the visual field.

- The presence of distractors is critical for the engagement of some, but not all, frontoparietal areas

In a further experiment, we investigated (Donner et al., 2003) whether the parietal cortex is also engaged in visual search without distractors. Two single object visual 
search tasks were matched in sensory stimulation and motor requirements, but were different in task difficulty. Differences between fMRI responses during both tasks were found within (predominantly left) AIPS and IPTO. Activation of PIPS and FEF was less reliable and failed to be significant in the group average (see Fig 6).

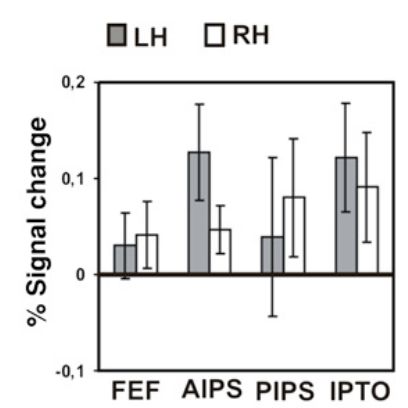

$\mathrm{N}=4$

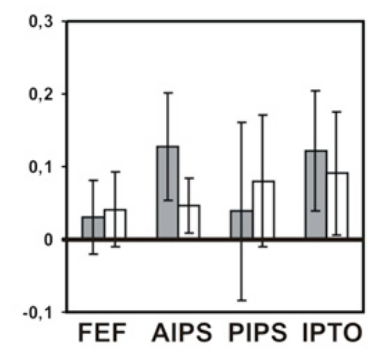

ROI

Figure 6. ROI responses during conjunction in the single object experiment. The signal is normalized to the mean of feature. Group averages are displayed with 95\% confidence intervals on the left and 99\% confidence intervals on the right. The amplitudes of differential responses were accepted as significant if the confidence interval did not include zero. In the group average, significant differential responses were found in AIPS and IPTO bilaterally and in the FEF and PIPS only in the right hemisphere. According to the 99\% confidence criterion, significant responses were restricted to AIPS and IPTO of both hemispheres in the group average.

Accordingly, these findings indicate that parts of PPC are engaged in attentional control even if a single peripheral object has to be identified. Neither the presence of inter-object competition (Reynolds \& Desimone, 1999) nor the necessity for distractor inhibition (Wojciulik \& Kanwisher, 1999) seems to be a prerequisite for their engagement. What kind of attention mechanism does PPC engagement in single object search reflect? At least, three types of mechanism are conceivable: (1) endogenous control of spatial attention shifts towards the peripheral object (Wolfe, 1994; Treisman \& Gelade, 1980; Corbetta et al., 1995), (2) prolonged maintenance of the attentional focus at the peripheral location during the identification of the feature conjunction (Chelazzi, 1999), and (3) the control of feature-based attention (Chelazzi, 1999; Wolfe, 1994; . Desimone \& Duncan, 1995; Grossberg et al., 1994).

Co-activation of multiple sub-regions appears to be a characteristic feature of parietal lobe function, complicating attempts to understand its functional organization
(Culham \& Kanwisher, 1999). By contrast, the present data point to a functional dissociation: AIPS and IPTO were consistently engaged while PIPS was not. Interestingly, Shulman and co-workers observed a predominantly left-hemispheric activation in AIPS during the delay of a non-spatial feature matching task (Shulman et al., 2002). This finding contrasts with the reliable predominance of right PPC in studies of spatial attention (Mesulam, 1999; Corbetta \& Shulman, 2002), but corresponds well with the present results.

We found that two sub-regions of the parietal cortex, AIPS and IPTO, are engaged in the attentional control of visual conjunction search irrespective of the presence of multiple distractors. By contrast, the engagement of another sub-region, PIPS, seems to presuppose the presence of distractors. Similar to non-spatial attention tasks, parietal activity during single object search predominates in the left hemisphere.

\section{Conclusion}

Functional neuroimaging studies of the visual selection process of visual search have began to yield important new constraints for functional and neural network models of this process. We focused on recent fMRI studies conducted in our lab, in which we compared fMRI activity between difficult and easy visual search tasks. The results suggest that (i) the human FEF and the three intraparietal sub-regions AIPS, PIPS and IPTO participate in the selection processes of visual search, (ii) that the participation of this 'frontoparietal attention network' does not depend on the necessity of feature conjunction, (iii) and that only a subset of parietal regions (AIPS and IPTO) but not the PIPS, participates in the control of visual search even in the absence of distractors. The results are thus generally consistent with models involving a spatially serial selection process in visual search. Furthermore they indicate that the group of frontoparietal areas commonly co-activated during attentionally demanding tasks seems to accommodate functionally specialized components. Subtle task variations as the ones used in the studies reviewed here might allow for revealing the differential functional repertoires of these cortical association areas. 


\section{References}

Allport, A. (1993). Attention and control: Have we been asking the wrong questions? A critical review of twenty five years. In: Meyer, D.E. \& Kornblum, S. (eds). Attention and Performance XIV. Hillsdale: Erlbaum, 182-217.

Andersen, R.A. \& Gnadt, J.W. (1989). Posterior parietal cortex. In: Wurtz, R.H. \& Goldberg, M.E, (eds). Neurobiology of Saccadic Eye Movements. Elsevier Science, 315-335.

Beauchamp, M.S., Petit, L., Ellmore, T.M., Ingeholm, J. \& Haxby, J.V. (2001). A parametric fMRI study of overt and covert shifts of visuospatial attention. $\mathrm{Neu}$ roimage $14,310-321$.

Bichot, N.P. \& Schall, J.D. (1999). Effects of similarity and history on neural mechanisms of visual selection. Nat. Neurosci. 2, 549-554.

Bichot, N.P., Cave, K.R. \& Pashler, H. (1999). Visual selection mediated by location: feature-based selection of noncontiguous locations. Percept Psychophys. 61(3), 403-23.

Bichot, N.P. \& Desimone, R. (2006). Finding a face in the crowd: parallel and serial neural mechanisms of visual selection. Prog Brain Res. 155,147-56.

Blaser, E., Pylyshyn, Z.W. \& Holcombe, A.O. (2000). Tracking an object through feature space. Nature. 408(6809),196-9.

Cave, K.R. \& Bichot, N.P. (1999). Visuospatial attention: beyond a spotlight model. Psychon Bull Rev. 6(2), 204-23.

Cave, K., Wolfe \& J.M. (1999). The psychophysical evidence for a binding problem in human vision. Neuron, 24, 11-17.

Chelazzi, L. (1999). Serial attention mechanisms in visual search: A critical look at the evidence. Psychol. Res. 62, 195-219.

Colby, C. L. \& Goldberg, M. E. (1999). Space and attention in parietal cortex. Annu. Rev. Neurosci. 22, 319 349.

Corbetta, M. \& Shulman, G.L. (2002). Control of goaldirected and stimulus-driven attention in the brain. Nat Rev Neurosci. 3(3), 201-15.

Corbetta, M., Shulman, G.L., Miezin, F.M. \& Petersen, S.E. (1995). Superior parietal cortex activation during spatial attention shifts and visual feature conjunction. Science 270, 802-805.
Corbetta, M., Akbudak, E., Conturo, T.E., Snyder, A.Z., Ollinger, J.M., Drury, H.A., Linenweber, M.R., Petersen, S.E., Raichle, M.E., Van Essen, D.C. \& Shulman, G.L. (1998). A common network of functional areas for attention and eye movements. Neuron 21, 761-773.

Corbetta, M. (1998). Frontoparietal cortical networks for directing attention and the eye to visual locations: identical, independent, or overlapping neural systems? Proc. Nat. Acad. Sci. USA 95, 831-838.

Courtney, S.M., Petit, L., Maisog, J.M., Ungerleider, L.G. \& Haxby, J.V. (1998) An area specialized for spatial working memory in human frontal cortex. Science, $279,13471351$.

Culham, J. \& Kanwisher, N. (1999). Neuroimaging of cognitive functions in human parietal cortex. Curr Opin Neurobiol 11, 157-163.

Desimone, R. \& Duncan, J. (1995). Neural mechanisms of selective visual attention. Annu Rev Neurosci MO 18, 193-222.

Donner, T., Kettermann, A., Diesch, E., Ostendorf, F., Villringer, A. \& Brandt, S.A. (2000). Involvement of the human frontal eye field and multiple parietal areas in covert visual selection during conjunction search. Eur. J. Neurosci. 12, 3407-3414.

Donner, T.H., Kettermann, A., Diesch, E., Ostendorf, F., Villringer, A. \& Brandt, S.A. (2002). Visual feature and conjunction searches of equal difficulty engage only partially overlapping frontoparietal networks. Neuroimage 15, 16-25.

Donner, T.H., Kettermann, A., Diesch, E., Villringer, A. $\&$ Brandt, S.A. (2003). Parietal activation during visual search in the absence of multiple distractors. Neuroreport. 14(17), 2257-61.

Duncan, J. (1984). Selective attention and the organization of visual information. J Exp Psychol Gen. 113(4), 501-17.

Duncan, J. \& Humphreys, G.W. (1989). Visual search and stimulus similarity. Psychol. Rev. 96, 433-458.

Duncan, J., Humphreys \& G., Ward, R. (1997). Competitive brain activity in visual attention. Curr Opin Neurobiol. 7(2), 255-61.

Felleman, D.J. \& Van Essen, D.C. (1991). Distributed hierarchical processing in the primate cerebral cortex. Cereb Cortex. 1(1), 1-47.

Findlay, J.M. (1997). Saccade target selection during visual search. Vis. Res., 37, 617-631. 
Friedman-Hill, S.R., Robertson, L.C. \& Treisman, A. (1995). Parietal contributions to visual feature binding: evidence from a patient with bilateral lesions. Science, 269, 853-855.

Gottlieb, J. P., Kusunoki, M. \& Golberg, M. E. (1998). The representation of visual salience in monkey parietal cortex. Nature. 391,481-484.

Groner, R. \& Groner M. T. (2000). The issue of control in sensory and perceptual processes: attention selects and modulates the visual input. In W.J. Perrig \& A. Grob (Eds.), pp.125-135. Control of human behavior, mental processes, and consciousness. Mahwah, N.J.: Lawrence Erlbaum.

Grossberg, S., Mingolla, E. \& Ross, W. D. (1994). A neural theory of attentive visual search: Interactions of boundary, surface, spatial,and object representations. Psychol. Rev. 101, 470-489.

Hamker. F.H. (2004). A dynamic model of how feature cues guide spatial attention. Vision Res. 44(5), 501521 .

Itti, L. \& Koch, C. (2001). Computational modelling of visual attention. Nature Rev. Neurosci., 2, 194-203.

Joseph, J.S., Chun, M.M. \& Nakayama, K. (1997). Attentional requirements in a preattentive' feature search task. Nature. 387(6635), 805-7.

Kastner, S. \& Ungerleider, L.G. (2000). Mechanisms of visual attention in the human cortex. Annu Rev Neurosci. 23, 315-41.

Kastner, S. \& McMains, S.A. (2007). Out of the spotlight: face to face with attention. Nat Neurosci. 10(11), 1344-5.

Koch, C. \& Ullman, S. (1985). Shifts in selective visual attention: towards the underlying neural circuitry. Hum Neurobiol., 4, 219-27.

Leonards, U., Sunaert, S., Van Hecke, P. \& Orban, G.A. (2000). Attention mechanisms in visual search - an fMRI study. J. Cogn. Neurosci. 12(S 2), 61-75.

Maunsell, J.H. \& Treue, S. (2006). Feature-based attention in visual cortex. Trends Neurosci. 29(6), 317-22. Epub 2006 May 11.

Mesulam. M-M. (1999). Spatial attention and neglect: parietal, frontal, and cingulate contributions to the mental representation and attentional targeting of salient extrapersonal events. Phil Trans Royal Soc Lond B $354,1325-1346$.
Mitchell, J.F., Stoner, G.R. \& Reynolds, J.H. (2004). Object-based attention determines dominance in binocular rivalry. Nature. 429(6990), 410-3.

Nakayama, K. \& Silverman, G.H. (1986). Serial and parallel processing of visual feature conjunctions. $\mathrm{Na}$ ture. 320(6059), 264-5.

Noton, D. \& Stark, L. (1971). Eye movements, and visual perception. Scientific American 224(6), 33-43.

O'Craven, K.M., Downing, P.E. \& Kanwisher, N. (1999). fMRI evidence for objects as the units of attentional selection. Nature. 401(6753), 584-7.

Paus, T. (1996). Location and function of the human frontal eye-field: a selective review. Neuropsychologia $34(6), 475-83$

Posner, M.I. \& Petersen, S.E. (1990). The attention system of the human brain. Annu. Rev. Neuroscience 13, 25-42. CrossRef, Medline, ISI

Reynolds, J. H. \& Desimone, R. (1999). The role of neural mechanisms of attention in solving the binding problem. Neuron 24, 19-29.

Robertson, L.C. (1998). Visuospatial attention and parietal function: their role in object perception. In Parasuraman, R. (eds). The Attentive Brain. Cambridge: MIT Press, 257-278.

Schall, J. D. \& Thompson, K. G. (1999). Neural selection and control of visually guided eye movements. Annu. Rev. Neurosci. 22, 2431-2459.

Shulman, G.L., d'Avossa, G., Tansy, A.P. \& Corbetta, M., (2002). Two attentional processes in the parietal lobe. Cereb coertex 12, 1124-1131.

Thompson, K.G. \& Bichot, N.P. (2005). A visual salience map in the primate frontal eye field. Prog Brain Res. 147, 251-62.

Treisman, A.M. \& Gelade, G. (1980). A featureintegration theory of attention. Cogn. Psychol. 12, 97136.

Treisman, A.M. (1996). The binding problem. Curr. Opin. Neurobiol. 6, 171-178.

Treue, S. (2003). Visual attention: the where, what, how and why of saliency. Curr Opin Neurobiol. 13(4), 428-32.

Wojciulik, E. \& Kanwisher, N. (1999). The generality of parietal involvement in visual attention. Neuron 23, 747-764. 
Wolfe, J.M. (1994). Guided search 2.0. A revised model of visual search. Psychonom. Bull. Rev. 1, 202-228.

Wolfe, J.M., Friedman-Hill, S.R. \& Bilsky, A.B. (1994) .Parallel processing of part-whole information in visual search tasks. Percept Psychophys. 55(5), 537-50.

Wolfe, J.M. (1998). Visual search. In Attention (H.

Pashler, Ed.), pp.13-73. Psychology Press, East Sussex. 